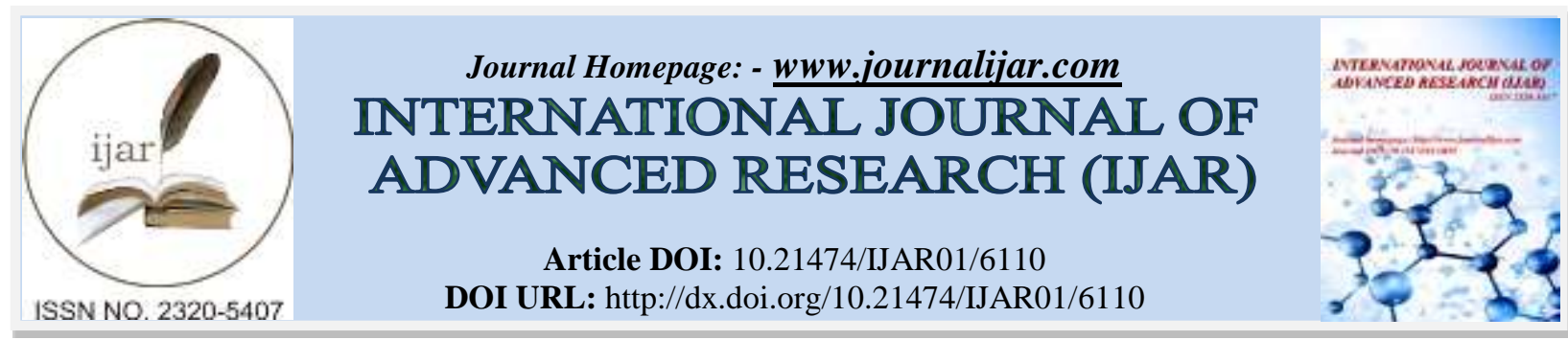

RESEARCH ARTICLE

\title{
TOPICAL APPLICATION OF FLUORIDE AND ITS ANTI-CARIOGENIC EFFECT.
}

\section{Omar T Mazyad ${ }^{1}$, Ahmed M El-marakby ${ }^{2,3}$, Yasser Refay Sorour ${ }^{4,5}$, Moath D Abo-ghannam ${ }^{1}$, Marwan M} Salem $^{1}$, Mohamed A Salamah ${ }^{1}$, Abdulkarim M Hawrani ${ }^{1}$ and Ashwag A Showail1 ${ }^{1}$.

1. Dental intern at Alfarabi colleges for Dentistry \& Nursing, Riyadh, Saudi Arabia.

2. Assistant Professor at Restorative Dental Science, Al-Farabi Colleges for Dentistry \& Nursing, Riyadh, Saudi Arabia.

3. Lecturer of Operative Dentistry, Faculty of Dentistry, Al-Azhar University, Assiut Branch, Egypt.

4. Lecturer of pediatric dentistry and dental public heath, Faculty of Dental Medicin, Al-Azhar University Assuiut branch. Egypt.

5. Assistant professor of pediatric dentistry, Head of Basic and Preventive sciences Department, Batterjee Medical College for Sciences and Technology, Faculty of Dentistry, Jeddah, Saudi Arabia.

\section{Manuscript Info}

(.........................

Manuscript History

Received: 20 October 2017

Final Accepted: 22 November 2017

Published: December 2017

\section{Keywords:-}

Fluoride compounds, Anti-cariogenic effect, Demineralization, Fluoride varnish.

\section{Abstract}

Most persons in industrial countries in addition to persons in some developing countries are suffering from dental caries (tooth decay) which is an infectious, multifactorial disease. Management of dental caries was shifted from "drill and fill" to "prevent and immune" after development of idea about the caries process. Many procedures and materials have been used for the preventive phase of dental caries. One of these materials was using the properties of fluoride with its anticariogenic properties. The incidence of dental caries (tooth decay) is reduced by using fluoride which slows, delays or arrests the progression of existingdental caries. This review article focused on different mechanism of action, types of topical applications and side effect of overdose use of fluoride compounds.

Copy Right, IJAR, 2017,. All rights reserved.

\section{Introduction:-}

Fluoride is a mineral that exists in all sources of the natural water and is the ionized form of the fluorine. Fluorine is ordinarily found in nature and reaches water sources by draining from soil and rocks into groundwater [1, 2]. Although fluoride compounds (e.g., calcium fluoride or sodium fluoride) represent a standard component of tooth enamel and bone, it can be also found in some plants. It is the most electro-negative element in the periodic table. Therefore, reactive with the strongest oxidizing action which binds to almost all other chemical elements. Many scientific papers reported the use of fluoride in improving oral health [3]. Fluoride likewise is created by some modern procedures that utilize the mineral apatite, a blend of calcium phosphate compound. In people, fluoride is primarily connected with calcified tissues (i.e., bones and teeth) as a result of its high affinity for calcium [4]. Fluoride's capacity to decrease the activity or even inverse the initiation and progression of dental caries is very much reported. The utilization of pre-adjusted fluoride in water for control of tooth decay began in 1945 and 1946 in the United States and Canada, at the point where fluoride concentration was balanced in drinking water providing four communities [5-8]. 
Regarding fluoride concentrations of community water supplies, In the 1940s and 1950s, The U.S. Public Health Service (PHS) developed recommendations. These recommendations assumed that the main source of fluoride is drinking water for most residents of the United States. As a result of the use of water fluoride in the prevention and control of tooth decay, various industrial fluoride products are developed, including toothpaste, rinsing mouth, dietary supplements, gels, foam or professionally applied or prescribed varnish. Furthermore, manufactured beverages, which constitute an expansion in the range of diets of many US residents, can contain a low concentration of fluoride, especially on the off chance that they are manufactured with fluorescent water. As such, US residents have more sources of fluoride now accessible than 50 years back. $[9,10]$

\section{Methodology:-}

We used scientific sites such as PubMed, Google Scholar and the Research Gate to get related articles about this subject. The research included specific keywords "topical application of fluoride, antimicrobial effect of fluoride, and fluoride as an antimicrobial agent" to find more articles related to the subject. We were more concerned about articles published in English only published from 1995 to 2017.

\section{Mechanism of Action of Fluoride:-}

The creation of newer toothpaste formulations has paralleled the increased understanding of the tooth decay process and how the fluoride does its action. The major belief of a continual dissolution of tooth surface has been replaced by the acceptance of an understanding of subsurface demineralization and re-mineralization [11]. When there is an imbalance between mineral gain and mineral loss, the demineralization occurs. These processes may be affected by fluoride in several ways. It is now widely accepted that fluoride has both systemic and topical modes of action [12]. The mechanism by which the fluoride prevents dental caries is summarized as follows:

\section{Protection Against Demineralization:-}

Fluoride interacted with the mineral component of teeth produces a fluoro-hydroxy-apatite (FHAP or FAP) mineral, where the hydroxyl group (OH-) is substituted by fluoride ion (F). As a result of this reaction, the hydrogen bond increased, a denser crystal lattice, and an overall decrease in solubility. As the amount of fluoride incorporation increase, the solubility decrease but rarely do we exceed several thousand parts per million of fluoride in the outer enamel [13].

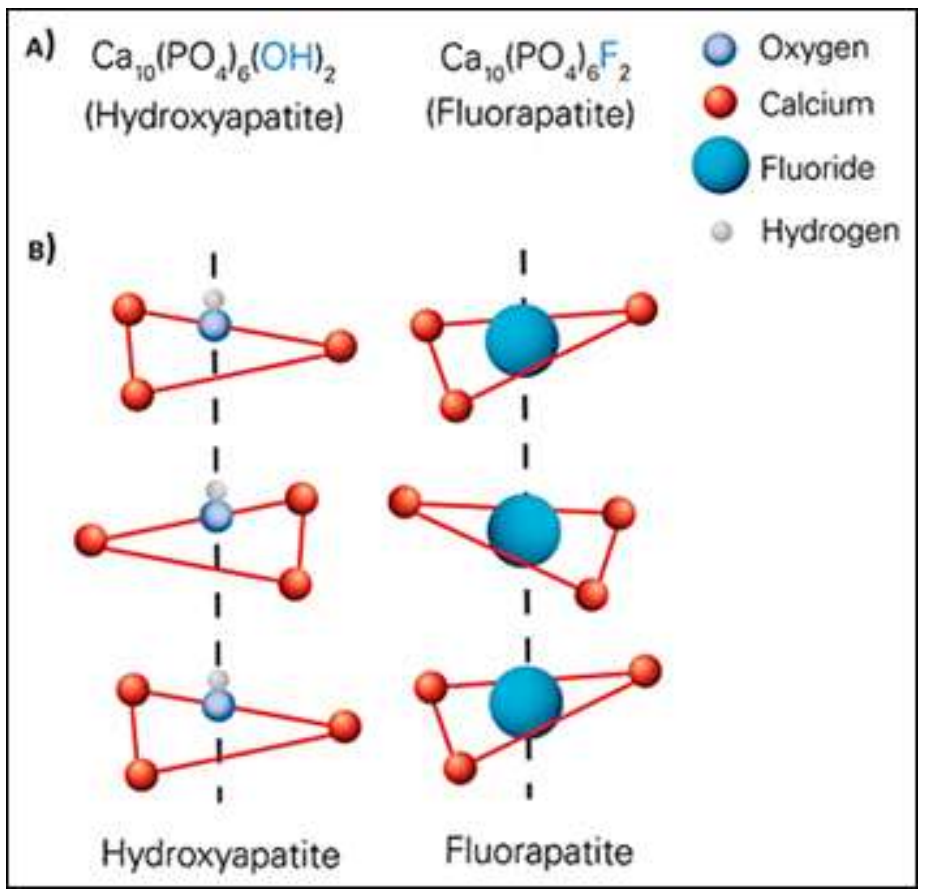

Figure 1:- Fluor-apatite Formation. 
1. The formation of fluor-apatite in the tooth enamel required the replacement of fluoride ions ( $\mathrm{F}-$ ) by hydroxyl ions $(\mathrm{OH}-)$ in hydroxyapatite

2. The replacement of hydroxide for fluoride in apatite crystal lattice [14].

Fluoride found in a pattern of solution can also affect the dissolution rate without changing the solubility of tooth minerals. In acidic medium, the dissolution rate of apatite decreased when the concentration of fluoride is less than $0.5 \mathrm{mg} / \mathrm{L}$ [15]. Absorption and/or ion conversation at the crystal surface is additionally included in this mechanism. In this way, the surface may act more like fluoro-hydroxy-apatite than hydroxy-apatite and have an alternate dissolution rate. At the point when the enamel dissolves, it may also contribute fluoride to the solution. The very low fluoride concentrations have been appeared to fundamentally decrease the dissolution rate of apatite [16]. Hence, presence of both types of fluorides either topically at the crystal surfaces or fluoride in the liquid phase concentrations during a cariogenic challenge are very important [17].

\section{Remineralization:-}

This is a process in which partially dissolved enamel crystals act as a substrate for mineral deposition from the solution phase that enables partial repair of the damaged crystals. Therefore, remineralization will face a bit of the demineralization, and an equilibrium will grow between the two processes. The carious lesion is the outcome of demineralization outweighing remineralization. One of the benefits of the demineralization / remineralization interplay is the creation of less soluble mineral in enamel [18]. This happens by dissolution of the more soluble calcium deficient magnesium containing carbonated apatite which marks up enamel when first formed. [19]. Too high of a supersaturation will effect the fast creation of calcium phosphate and block the surface pores of enamel. This precipitation reduces the diffusion of calcium, phosphate, and fluoride into the interior of the lesion, which can result in lesion arrestment rather than lesion repair [20]. The interior of the lesion is partially saturated concerning HAP and can become supersaturated concerning FAP, even if minimal levels of fluoride are present or diffuse into the lesion. The use of low concentration fluoride products, such as dentifrices on a daily basis, will help maintain this favorable saturation. Thus, remineralization of the lesion may result in the repair of the existing lesion with less soluble mineral and render this portion of the tooth less susceptible to future episodes of demineralization (Figure 2). This is probably one of the most important modes of action of fluoride. At relatively low concentration, Fluoride may also interact with the oral bacteria to limit plaque acid production. Several mechanisms have been anticipated to account for this result. One is the well-known interaction of fluoride with the enzyme enolase which could reduce acid production directly. There is also an indirect effect on the phosphotransferase system (PTS) pathway that decreases the amount of sugar entering the cell by limiting phosphoenolpyruvate (PEP).

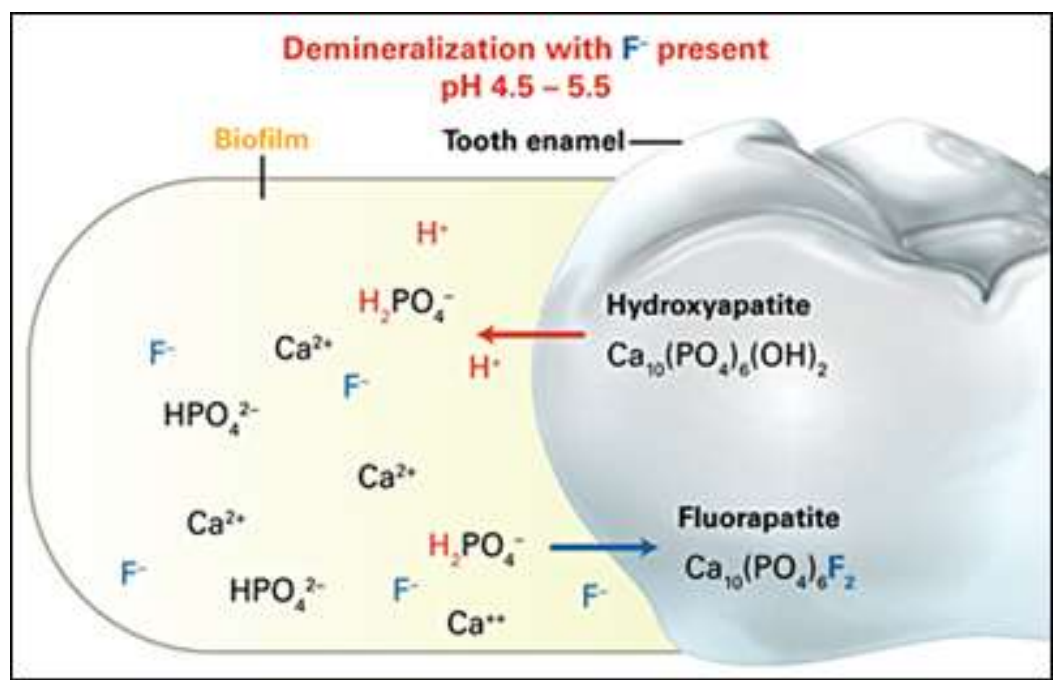

Figure (2):- Fluoride Reactivity.

In the plaque biofilm, the bacteria converted the carbohydrates into acids. In an acidic medium (pH less than 5.5), the biofilm fluid becomes under-saturated with phosphate ion and enamel dissolves to restore balance. When fluoride ions are present, fluor-apatite is incorporated into demineralized enamel and leading to demineralization inhibition [21]. 
Topical application of fluoride:-

Self-Applied : Fluoride Toothpaste:-

Fluoride-containing toothpaste is the most commonly used form of self-applied fluoride worldwide [23]. Fluoride in toothpaste is taken up directly by the dental plaque and demineralized enamel and also increases the concentration of fluoride in saliva [22]. The fluoride concentration increases in saliva 100- to 1,000-fold by brushing with fluoride toothpaste. This concentration returns to baseline levels within 1 to 2 hours [23]. For the vast majority (kids, young people, and grown-ups) brushing twice per day with a fluoride toothpaste when get up in the morning and before going to bed is highly recommended [22]. Children's brushing ought to be administered to guarantee that they utilize the proper measure of toothpaste. For youngsters more youthful than three years, parents and caregivers should start brushing kids' teeth when they begin to come into the mouth by utilizing fluoride toothpaste in a sum portrayed as close to spread or on the other hand as the span of a grain of rice [23]. For kids 3 to 6 years old, parents and caregivers ought to administer close to a pea-sized measure of fluoride toothpaste [23].

\section{Fluoride Mouth rinse or Gels:-}

Fluoride mouth rinse is a concentrated solution proposed for every day or week after week utilize and intended to be washed and spit out [22]. The most widely recognized fluoride compound used as a part of mouth rinse is sodium fluoride [22]. The fluoride from mouth rinse is held in dental plaque and saliva and averts tooth decay [22, 23]. An over-the-counter solution of $0.05 \%$ sodium fluoride $(230 \mathrm{ppm}$ fluoride) for day by day flushing are accessible for use by people more established than six years of age [22, 24, 25]. The use in people more youthful than six years old isn't suggested in light of the danger of fluorosis if the wash is repeatedly gulped [23, 25]. Higher quality mouth rinses (e.g., $0.2 \%$ impartial sodium fluoride to be utilized once per week) for those at high danger of tooth rot must be recommended by a dental specialist or physician [25]. Solutions of $0.2 \%$ sodium fluoride $(920$ ppm fluoride) are likewise utilized as a part of regulated, school-based week by week washing programs [22, 23, 24].

\section{Professionally Applied:-}

\section{Fluoride Mouth rinse, Gels, or Foams:-}

Professionally applied fluorides are as a gel, froth or wash, and are applied by a dental expert during dental visits [22]. Professionally applied fluorides are more concentrated than the self-applied ones (e.g., 1.23\% fluoride particle [12,300 ppm]), and like this is not required frequently. An early study of Dean et al., revealed that fluoride take-up by dental enamel increased in an acidic medium, fluoride gel is frequently figured to be highly acidic $(\mathrm{pH}$ about 3.0) [7]. Products accessible in the U.S. incorporate gels of acidulated phosphate fluoride $(1.23 \%$ [12,300 ppm] fluoride), as $2 \% \%$ neutral sodium fluoride compounds (containing 9,000 ppm fluoride), and as gels or foams of sodium fluoride $(0.9 \%$ [9,040 ppm] fluoride). In a dental office, fluoride gel is applied for 1 to 4 minutes [22, 23, 24]. Since these applications are moderate infrequent, generally at three months to 1-year interims, fluoride gel postures little hazard for dental fluorosis, even among patients less than six years of age. Routine utilization of professionally applied fluoride gel or foam likely gives advantage just to people at high risk for caries, particularly the individuals who do not consume fluoridated water and brush every day with fluoride toothpaste [22, 23].

\section{Fluoride-Containing Prophylaxis Paste:-}

The fluoride-containing paste is routinely utilized with dental prophylaxis. The abrasive paste, which contains 4,000 to 20,000 ppm fluoride, may reestablish the concentration of fluoride in the surface layer of enamel removed by cleaning, however it is not a sufficient substitute for fluoride gel or varnish in treating people at high risk for tooth decay [23]. Fluoride prophylaxis paste alone isn't considered by the U.S. Food and Drug Administration (FDA) or ADA a powerful technique to diminish tooth decay $[22,26]$.

\section{Fluoride Varnish:-}

Varnishes are found as sodium fluoride (2.26\% [22,600 ppm] fluoride) or difluorsilane (0.1\% [1,000 ppm] fluoride) preparations. An ordinary application requires 0.2 to $0.5 \mathrm{~mL}$, leading to a total fluoride particle utilization of around 5 to $11 \mathrm{mg}$. Highly concentrated fluoride varnish is painted by dental or other medical professionals especially when applied onto the teeth and sets when it comes into contact with saliva [22, 24, 25]. Fluoride varnish is not is not intended to adhere forever; this strategy holds a high concentration of fluoride in a small amount of material in close contact with the teeth for a few hours. Varnishes must be reapplied at regular intervals with no less than two applications for each year required for the sustained benefit. Although it is not right now cleared for advertising by the FDA as an anti-caries agent, fluoride varnish has been utilized for this reason in Canada and Europe since the 1970s [22, 23]. 


\section{Silver Diamine Fluoride:-}

Silver diamine fluoride (SDF) is a transparent fluid that at $\mathrm{pH} 10$ is $24.4 \%$ to $28.8 \%$ (weight/volume) silver and $5.0 \%$ to $5.9 \%$ fluoride [24]. The FDA has characterized SDF as a Class II therapeutic device; it is cleared for use in the treatment of tooth sensitivity and should be applied by professionals. in spite of a few items are industrially accessible in different nations, right now, Advantage Arrest ${ }^{\mathrm{TM}}$ (Elevate Oral Care, L.L.C.) is the main financially accessible SDF item for dental use in the U.S [25]. There have been reports of the utilization of SDF in caries control and administration, in spite of the fact that it is not particularly marked for use for this sign (i.e., "off-name utilize"). Likely a consequence of its fluoride content, when applied to carious inflammation, SDF has been appeared to decrease caries danger of the adjacent tooth surface [25-28]. SDF has likewise demonstrated viability in the administration of root caries in the elderly. It likely has extra appropriateness as an interim approach for overseeing dangerous caries in people currently unable to tolerate more involved dental therapy [29-32]. Single use of SDF has been accounted for to be lacking for the supported benefit [33]. Its potential drawbacks incorporate an apparently unpleasant metallic taste, the potential to disturb gingival and mucosal surfaces, and the characteristic dark coloring of the tooth surfaces to which it is applied [27].

Fluoride side effects and safety:-

The amounts of fluoride which was added to public water supplies and used in toothpaste and mouthwashes, and applied by dentists are safe for most people. Low dosages (up to $20 \mathrm{mg}$ daily of elemental fluoride) of supplemental fluoride taken by mouth is being safe for most people. Higher doses are unsafe and can cause bone and tendons and muscle weakness. Also it has bad effects on the nervous system. In children -before their permanent teeth come through- high fluoride concentrations can cause a change in the tooth color. Toothpaste and fluoride washes ought not to be swallowed routinely, especially by youngsters. It is a smart thought to ensure that kids under six years old utilize just a pea-sized amount of fluoride-containing toothpaste, just if they swallow some [34].

\section{Special precautions and warnings:-}

Pregnancy and lactation: during pregnancy and lactation the level of fluoride is safe when taken in doses below the acceptable upper intake level (UL) of $10 \mathrm{mg}$ daily of elemental fluoride and when directly applied to the teeth in toothpastes and mouthwashes. while higher doses are unsafe and can cause bone and tendons and muscle weakness, also it has bad effects on the nervous system[34].

\section{Conclusion:-}

Fluorine is ordinarily found in nature and reaches water sources by draining from soil and rocks into groundwater. Fluorine is the most electronegative element in the periodic table, and consequently, it is the most reactive one. Fluoride is used to reduce dental caries by affecting the subsurface demineralization and re-mineralization. Self and professional topical application of fluoride have anticariogenic effect.

\section{References:-}

1. Bratthall D, Hänsel Petersson G, Sundberg H. Reasons for the caries decline: what do the experts believe? Eur J Oral Sci 1996;104:416--22.

2. McGrady MG, Ellwood RP, Pretty IA. Why fluoride? Dent Update 2010; 37(9):595-8, 601-2.

3. Haugejorden O, Nord A, Klock KS. Direct evidence the major role of fluoride dentifrices in the caries decline. A 6-year analytical cohort study. Acta Odontol Scand. 1997;55(3):173-180.

4. Kohn, W. G., Maas, W. R., Malvitz, D. M., Presson, S. M., \& Shaddix, K. K. (2001). Recommendations for using fluoride to prevent and control dental caries in the United States.

5. Blaney JR, Tucker WH. The Evanston Dental Caries Study. II. Purpose and mechanism of the study. J Dent Res 1948;27:279--86.

6. Ast DB, Finn SB, McCaffrey I. The Newburgh-Kingston Caries Fluorine Study. I. Dental findings after three years of water fluoridation. Am J Public Health 1950;40:716--24.

7. Dean HT, Arnold FA, Jay P, Knutson JW. Studies on mass control of dental caries through fluoridation of the public water supply. Public Health Rep 1950;65:1403--8.

8. Hutton WL, Linscott BW, Williams DB. The Brantford fluorine experiment: interim report after five years of water fluoridation. Can J Public Health 1951;42:81--7.

9. Pao EM. Changes in American food consumption patterns and their nutritional significance. Food Technol 1981;35:43--53. 
10. Heller KE, Sohn W, Burt BA, Eklund SA. Water consumption the United States in 1994--1996 and implications for water fluoridation policy. J Public Health Dent 1999;59:3--11.

11. Silverstone LM. Remineralization phenomena. Caries Res. 1977;11 Suppl 1:59-84.

12. Wefel JS. Mechanisms of action of fluoride - Pediatric Dentistry: Scientific Foundations and Clinical Practice. Ray Stewart(Ed). St. Louis, MO. Mosby. 1982.

13. Moreno EC, Kresak M, Zahradnik RT. Physicochemical aspects of fluoride-apatite systems relevant to the study of dental caries. Caries Res. 1977;11 Suppl 1:142-171.

14. Posner AS. The mineral of bone. Clin Orthop Relat Res. 1985 Nov;(200):87-99.

15. Christoffersen MR, Christoffersen J, Arends J. Kinetics of dissolution of calcium hydroxyapatite: VII. The effect of fluoride ions - Journal of Crystal Growth. 1984;67(1):107-114. Accessed April 10, 2017.

16. Wong L, Cutress TW, Duncan JF. The influence of incorporated and adsorbed fluoride on the dissolution of powdered and pelletized hydroxyapatite in fluoridated and non-fluoridated acid buffers. J Dent Res. 1987 Dec;66(12):1735-1741. doi: 10.1177/00220345870660120801.

17. Shellis RP, Duckworth RM. Studies on the cariostatic mechanisms of fluoride. Int Dent J. 1994 Jun;44(3 Suppl 1):263-273.

18. Wefel JS, Dodds MWJ. Oral biologic defenses and the demineralization and remineralization of teeth - Primary Preventive Dentistry, 5th edition. Norman Harris (Ed). Stamford, CN. Appleton and Lange. 1999. 271-298.

19. Silverstone LM, Wefel JS. The effect of remineralization on artificial caries-like lesions and their crystal content - Journal of Crystal Growth. 1981;53(1):148-159. Accessed April 10, 2017.

20. Silverstone LH. Fluorides and remineralizations - Clinical Uses of Fluorides (Current Problems in Clinical Dentistry). Stephen HY Wei (Ed). London. 1986. 153-175.

21. Cury JA, Tenuta LM. Enamel remineralization: controlling the caries disease or treating early caries lesions? Braz Oral Res. 2009;23 Suppl 1:23-30.

22. Centers for Disease and Prevention. Other Fluoride Products. U.S. Department of Health and Human Services. Accessed August 3, 2017.

23. Recommendations for using fluoride to prevent and control dental caries in the United States. Centers for Disease Control and Prevention. MMWR Recomm Rep 2001;50(Rr-14):1-42.

24. Adair SM. Evidence-based use of fluoride in contemporary pediatric dental practice. Pediatr Dent 2006;28(2):133-42; discussion 92-8.

25. Clark MB, Slayton RL. Fluoride use in caries prevention in the primary care setting. Pediatrics 2014;134(3):626-33.

26. Weyant RJ, Tracy SL, Anselmo TT, et al. Topical fluoride for caries prevention: executive summary of the updated clinical recommendations and supporting systematic review. J Am Dent Assoc 2013;144(11):1279-91.

27. Mei ML, Lo EC, Chu CH. Clinical Use of Silver Diamine Fluoride in Dental Treatment. Compend Contin Educ Dent 2016;37(2):93-8; quiz100.

28. Llodra JC, Rodriguez A, Ferrer B, et al. Efficacy of silver diamine fluoride for caries reduction in primary teeth and first permanent molars of schoolchildren: 36-month clinical trial. J Dent Res 2005;84(8):721-4.

29. Li R, Lo EC, Liu BY, Wong MC, Chu CH. Randomized clinical trial on arresting dental root caries through silver diammine fluoride applications in community-dwelling elders. J Dent 2016.

30. Zhang W, McGrath C, Lo EC, Li JY. Silver diamine fluoride and education to prevent and arrest root caries among community-dwelling elders. Caries Res 2013;47(4):284-90.

31. Hendre AD, Taylor GW, Chavez EM, Hyde S. A systematic review of silver diamine fluoride: Effectiveness and application in older adults. Gerodontology 2017.

32. Giusti L, Steinborn C, Steinborn M. Use of silver diamine fluoride for the maintenance of dental prostheses in a high caries-risk patient: A medical management approach. J Prosthet Dent 2017.

33. Horst JA, Ellenikiotis H, Milgrom PL. UCSF Protocol for Caries Arrest Using Silver Diamine Fluoride: Rationale, Indications and Consent. J Calif Dent Assoc 2016;44(1):16-28.

34. Rios D, Magalhães AC, Polo RO, Wiegand A, Attin T, Buzalaf MA: The efficacy of a highly concentrated fluoride dentifrice on bovine enamel subjected to erosion and abrasion. J Am Dent Assoc 2008;139:1652-1656. 